\title{
Espécies de moscas ectoparasitas (Diptera, Hippoboscoidea) de morcegos (Mammalia, Chiroptera) no estado do Maranhão
}

\author{
Paulo Adriano Dias ${ }^{1}$, Ciro Líbio Caldas dos $\operatorname{Santos}^{2}$, Fernanda Souto Rodrigues ${ }^{2}$, Luciana Cordeiro Rosa², Keliane Silva \\ Lobato $^{2}$ \& José Manuel Macário Rebêlo ${ }^{1,2}$
}

\begin{abstract}
${ }^{1}$ Mestrado em Biodiversidade e Conservação, PPGBC/Departamento de Biologia-UFMA, Av dos portugueses, SN. Campus do Bacanga, 65040-080 São Luis-MA. diaspad@ hotmail.com; macariorebelo@uol.com.br

${ }^{2}$ Laboratório de Entomologia e Vetores/Departamento de Patologia/Universidade Federal do Maranhão - LEV/DPATOL/UFMA; Av. dos Portugueses S/N, Campus do Bacanga, 65000-000 São Luís-MA. cirolb@yahoo.com.br; nanda_eco@yahoo.com.br; lu_se_ama@yahoo.com.br;

keliane_lobato@yahoo.com.br
\end{abstract}

\begin{abstract}
Parasitic flies (Diptera, Hippoboscoidea) on bats (Mammalia, Chiroptera) in Maranhão state. This list of species is the first study dealing with bat flies in the Maranhão State. The work was done at seven different areas in the cities Bacabeira, São Luis, Santa Inês and Tufilândia. The bats were captured in mist nets and the parasites were collected with tweezers. A total of 559 bat flies belonging to 25 species, (22 species of the family Streblidae and 2 of Nycteribiidae), were colleted from 118 bats belonging to 22 species, 13 genera and 4 families. Trichobius joblingi Wenzel, 1966 was the most frequent species with $28.7 \%$ of the collected individuals. This study registered a high number of species of bat flies in the state, evidencing the importance of the area for studies of biodiversity, as well as aspects related to host-parasite relationships.
\end{abstract}

KEYWORDS. Biodiversity; host; inventory; Nycteribiidae; Streblidae.

RESUMO. Espécies de moscas ectoparasitas (Diptera, Hippoboscoidea) de morcegos (Mammalia, Chiroptera) no estado do Maranhão. Esta lista preliminar de espécies de moscas ectoparasitas de morcegos é o primeiro estudo com esse grupo no Estado do Maranhão. O levantamento foi realizado em sete localidades nos municípios Bacabeira, São Luis, Santa Inês e Tufilândia. Os morcegos foram capturados em redes de neblina e os parasitas retirados destes com pinças. No total foram capturadas 559 moscas pertencentes a 25 espécies (11 gêneros), sendo 22 espécies da família Streblidae e duas da Nycteribiidae. Trichobius joblingi Wenzel, 1966 foi a espécie mais freqüente, representando $28.7 \%$ dos indivíduos coletados. As moscas encontravam-se infestando 118 morcegos pertencentes a 22 espécies, 13 gêneros e 4 famílias. Este estudo registrou uma alta riqueza de espécies de moscas e de morcegos no estado, evidenciando a importancia da região para estudos em biodiversidade, bem como aspectos relacionados à relação parasito-hospedeiro.

PALAVRAS-CHAVE. Biodiversidade; hospedeiro; inventário; Nycteribiidae; Streblidae.

O Estado do Maranhão por sua extensão territorial e posição estratégica de confluência dos biomas Amazônia, Cerrado e Caatinga, e ainda por apresentar formações típicas como a Mata de Cocais e Baixada, aparece no cenário nacional como uma das áreas de maior diversidade animal e vegetal (Ab’Saber 1977; Muniz 2006).

Vários grupos de insetos vêm sendo estudados no Maranhão, especialmente os dípteros de importância médicoveterinária. Dentre eles destacam-se os dípteros nematóceros hematófagos vetores de doenças, incluindo os anofelinos (culicídeos), flebotomíneos (psicodídeos) e os maruins (ceratopogonídeos), cuja composição faunística é relativamente bem conhecida no Estado (Rebêlo et al. 1996; Pereira e Rebêlo 2000).

Contudo, apesar da importância do estado do Maranhão no tocante à diversidade de ecossistemas e ao volume de estudos envolvendo insetos, até o presente momento, não existe nenhum estudo relatado na literatura, sobre as moscas ectoparasitas de morcegos, o segundo grupo de mamíferos mais diversificado.

As moscas ectoparasitas de morcegos pertencem à
Superfamília Hippoboscoidea estando distribuídas entre duas famílias, exclusivamente ectoparasitas de morcegos, Nycteribiidae e Streblidae (Marshall 1982).

A família Nycteribiidae caracteriza-se por apresentar indivíduos com mesonoto reduzido e despigmentado e, as pleuras deslocadas para a face dorsal devido à expansão do esterno torácico, aparentando aspecto acariforme (Graciolli 2004). Atualmente, a família compreende 274 espécies, incluídas em 11 gêneros e três subfamílias: Nycteribiinae, Cyclopodiinae e Archinycteribiinae (Maa 1965; Graciolli \& Dick 2006). No continente americano são encontrados dois gêneros, Basilia Miranda - Ribeiro, 1903 e Hershkovitzia Guimarães e D'andretta, 1956. No Brasil, são registradas 24 espécies de Basilia Miranda-Ribeiro 1903 e três espécies de Hershkovitzia Guimarães \& D’Andretta 1956 (Graciolli et al. 2007).

A família Streblidae é formada por espécies ápteras, braquípteras e aladas sendo encontrada em todas as regiões biogeográficas, principalmente, em áreas tropicais (Wenzel 1970). Atualmente está dividida em cinco subfamílias, Nycteriboscinae, Ascopterinae, Trichobiinae, Streblinae e Nycterophiliinae, sendo as três últimas exclusivas do Novo 
Mundo, representadas por 33 gêneros e 230 espécies (Guerrero 1997; Dick e Graciolli 2006). Segundo Guerrero (1997) a família Streblidae é relativamente bem conhecida no continente americano, tendo-se registro de várias espécies distribuídas no Panamá (69 espécies), Colômbia (54 espécies), Venezuela (119 espécies), Peru (59) e para o Brasil são reconhecidas cerca de 70 espécies (Prevedello et al.2005).

Pioneiro para o grupo no Maranhão este trabalho surge como ferramenta de produção de conhecimento sobre a biodiversidade do Estado, como também poderá gerar subsídios para possíveis estudos em ecologia de parasitas, filogenia e transmissão de doenças. Para tanto este visou realizar o levantamento das espécies de moscas ectoparasitas de morcegos que ocorrem no Estado do Maranhão, com o intuito de elaborar uma listagem e caracterizar as relações específicas estabelecidas entre os dois grupos faunísticos.

\section{MATERIALE MÉTODOS}

Descrição da área. Neste trabalho as moscas e os morcegos foram amostrados em quatro localidades no município de São Luís escolhidas por apresentar habitats diferentes entre si: 1 A comunidade de Quebra-Pote $\left(02^{\circ} 41^{\prime} \mathrm{S}\right.$ e $44^{\circ} 12^{\prime} \mathrm{W}$ ) com vegetação secundária, mata de cocais, mangue e brejo de buriti; 2 - Parque Ambiental da empresa ALUMAR (02 $44^{\circ} \mathrm{S}$ e $44^{\circ} 17^{\prime} \mathrm{W}$ ) em fragmentos de floresta amazônica com vegetação semelhante à de várzea, mata de terra firme e campo abandonado; 3 - uma área de borda de mangue pertencente ao Terminal Portuário do Itaqui - TEC $\left(02^{\circ} 34^{\prime} \mathrm{S}\right.$ e $\left.44^{\circ} 21^{\prime} \mathrm{W}\right)$ e 4 - no Parque Estadual do Bacanga - PEB ( $\left.2^{\circ} 34^{\prime} \mathrm{S} 44^{\circ} 15^{\prime} \mathrm{W}\right)$ caracterizado por mata amazônica secundária com campos abandonados. No interior do Estado foram realizadas coletas nos municípios de Bacabeira ( $2^{\circ} 57^{\prime} \mathrm{S} 44^{\circ} 24^{\prime} \mathrm{W}$ ) em Borda de mangue, Capoeira de babaçu, fragmento de mata e campo alagadiço; em Santa Inês ( $3^{\circ} 39^{\prime}$ S 45 23'W) e Tufilândia (341'S $45^{\circ} 35^{\prime} \mathrm{W}$ ) em Bosque peridomiciliar.

Captura dos morcegos e coleta das moscas. No período de maio de 2006 a junho de 2007 foram realizadas 16 noites de coletas, sendo 13 na Ilha de São Luís e três no interior do Estado (Fig. 1). As coletas foram realizadas das $18 \mathrm{~h} 00$ às $00 \mathrm{~h} 00$ resultando num esforço de captura de 16,14.h. $\mathrm{m}^{2}$ de rede.

A captura dos morcegos foi realizada com a utilização de redes de neblina, com tamanho de $10 \mathrm{~m} \times 2,5 \mathrm{~m}$, armadas ao nível do solo por um período de seis horas após o crepúsculo, utilizando a metodologia sugerida por Pacheco (2005).

Todos os morcegos capturados foram ainda em campo identificados seguindo a chave de Burton e Engstron (2001). Estes foram biometrados e vistoriados a olho nú por dois observadores ao mesmo tempo. Quando não puderam ser identificados em campo foram fixados em alcool $70 \%$, e depositados na coleção da Universidade Estadual do Maranhão para posterior estudo.

Todas as moscas ectoparasitas encontradas foram coletadas na superfície corporal dos morcegos com o auxílio de pinças de ponta fina e pincéis embebidos em álcool, fixadas em álcool a

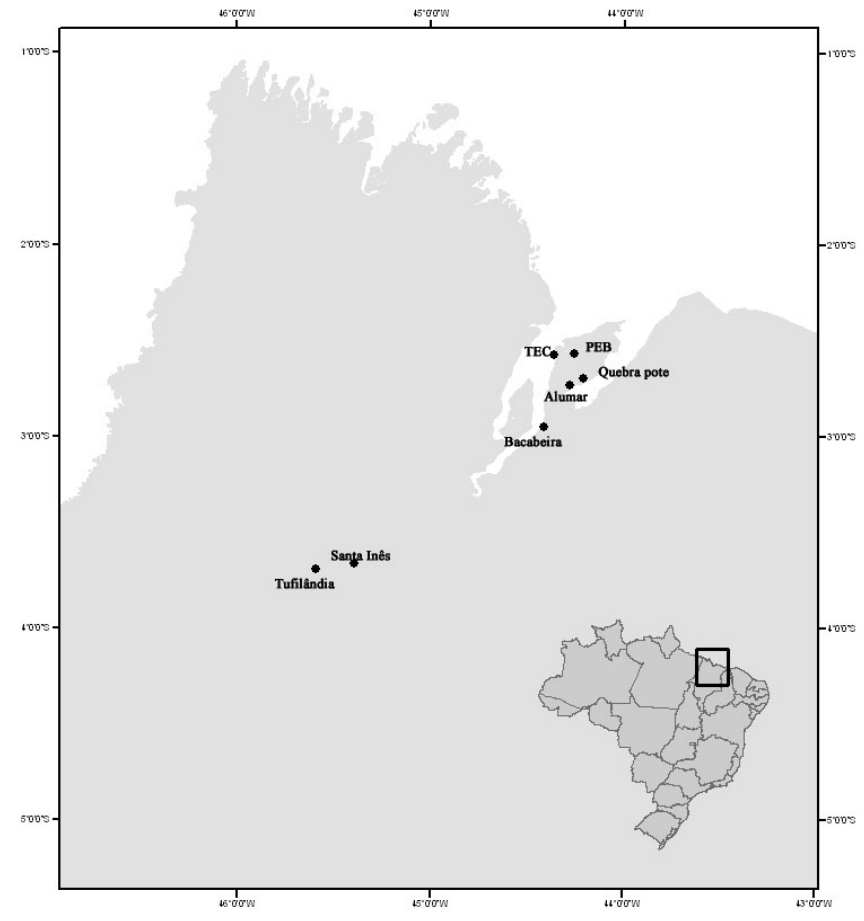

Fig. 1. Localidades amostradas para moscas ectoparasitas de morcegos no Estado do Maranhão.

$70 \%$ e acondicionadas em frascos individuais, separadamente para cada hospedeiro.

Identificação das moscas. A triagem dos dípteros da família Streblidae foi realizada no Laboratório de Entomologia e Vetores (LEV) do Departamento de Patologia da Universidade Federal do Maranhão, e os espécimens foram identificados pelo professor Gustavo Graciolli da Universidade Federal do Mato Grosso do Sul. Os espécimes coletados encontram-se despositados nas coleções das duas instituições.

A nomenclatura adotada para os estreblídeos segue a sugerida por Dick \& Graciolli (2006), para a família Nycteribiidae a sugerida por Graciolli \& Dick (2006) enquanto que a utilizada para os morcegos a sugerida por Reis et al. (2007). Alguns indivíduos da família Nycteribiidae não puderam ser identificados ao nível de espécie, ou por falta de material de referência ou por constituir-se de espécimes, cujo sexo ou estado, não serviam para caracterizar a espécie.

\section{RESULTADOS EDISCUSSÃO}

Nas sete localidades estudadas foi coletado um total de 559 moscas de 25 espécies, sendo 556 Streblidae (23 espécies pertencentes a 10 gêneros), e três Nycteribiidae todas do gênero Basilia (Tab. I).

As moscas foram capturadas sobre 118 morcegos infestados, pertencentes a 21 espécies, 13 gêneros e 4 famílias (Tab. II).

Foram ainda registradas sem infestação as espécies de morcegos: Saccopteryx bilineata (Temminck, 1838), Noctilio leporinus (Linnaeus, 1758), Macrophyllum macrophyllum 
Tabela I. Lista e freqüência relativa das espécies de moscas ectoparasitas registradas para o Estado do Maranhão, nas localidades: Reserva Ambiental da ALUMAR (ALUMAR); Município de Bacabeira (BAC); Terminal Portuário do Itaqui (TEC); Parque Estadual do Bacanga (PEB); povoado Quebra Pote (QP); Município de Santa Inês (SI); Município de Tufilândia (TUF).

\begin{tabular}{|c|c|c|c|c|c|c|c|c|}
\hline \multirow[t]{2}{*}{ Táxon } & \multicolumn{7}{|c|}{ Localidades } & \multirow[t]{2}{*}{$\%$} \\
\hline & ALUMAR & BAC & TEC & PEB & QP & SI & TUF & \\
\hline \multicolumn{9}{|l|}{ STREBLIDAE } \\
\hline \multicolumn{9}{|l|}{ Subfamília Trichobiinae } \\
\hline Aspidoptera falcata Wenzel, 1976 & & & & & $\mathrm{X}$ & & & 1,25 \\
\hline Aspidoptera phyllostomatis (Perty, 1833) & & & & $\mathrm{X}$ & $\mathrm{X}$ & & & 2,32 \\
\hline Mastoptera minuta (Costa Lima, 1921) & & $\mathrm{X}$ & & $\mathrm{X}$ & $\mathrm{X}$ & & $\mathrm{X}$ & 16,1 \\
\hline Megistopoda aranea (Coquillett, 1899) & & $\mathrm{X}$ & $\mathrm{X}$ & $\mathrm{X}$ & $\mathrm{X}$ & & & 8,58 \\
\hline Megistopoda proxima (Séguy, 1926) & & & & & $\mathrm{X}$ & & & 1,43 \\
\hline Paradyschiria parvula Falcoz, 1931 & & $\mathrm{X}$ & & & & & & 3,93 \\
\hline Paratrichobius longicrus (Miranda Ribeiro, 1907) & & & & & & $\mathrm{X}$ & & 0,17 \\
\hline Speiseria ambigua Kessel, 1925 & $\mathrm{X}$ & & $\mathrm{X}$ & $\mathrm{X}$ & $\mathrm{X}$ & & & 5,18 \\
\hline Stizostrebla longirostris Jobling, 1939 & & & & & $\mathrm{X}$ & & & 0,89 \\
\hline Trichobioides perspicillatus (Pessôa \& Galvão, 1937) & & $\mathrm{X}$ & $\mathrm{X}$ & & $\mathrm{X}$ & & & 2,32 \\
\hline Trichobius costalimai Guimarães, 1938 & $\mathrm{X}$ & $\mathrm{X}$ & $\mathrm{X}$ & $\mathrm{X}$ & $\mathrm{X}$ & & & 13,05 \\
\hline Trichobius diaemi Wenzel, 1976 & & & & & & & $\mathrm{X}$ & 6,61 \\
\hline Trichobius dugesii Townsend, 1891 & & & & & $\mathrm{X}$ & & & 0,71 \\
\hline Trichobius dugesioides phyllostomus Guerrero, 1998 & & & & & $\mathrm{X}$ & & & 0,17 \\
\hline Trichobius joblingi Wenzel, 1966 & $\mathrm{X}$ & $\mathrm{X}$ & $\mathrm{X}$ & $\mathrm{X}$ & $\mathrm{X}$ & $\mathrm{X}$ & & 28,62 \\
\hline Trichobius longipes (Rudow, 1871) & & & & & $\mathrm{X}$ & & $\mathrm{X}$ & 1,78 \\
\hline Trichobius parasiticus Gervais, 1844 & & $\mathrm{X}$ & & & & & & 0,17 \\
\hline \multicolumn{9}{|l|}{ Subfamília Streblinae } \\
\hline Strebla diaemi Wenzel, 1966 & & & & & & & $\mathrm{X}$ & 1,61 \\
\hline Strebla galindoi Wenzel, 1966 & & $\mathrm{X}$ & & & & & & 1,96 \\
\hline Strebla guajiro (García \& Casal, 1965) & $X$ & & $\mathrm{X}$ & & $\mathrm{X}$ & & & 1,07 \\
\hline Strebla hertigi Wenzel, 1966 & & $\mathrm{X}$ & $\mathrm{X}$ & & $\mathrm{X}$ & & & 0,53 \\
\hline Strebla sp. & & & & $\mathrm{X}$ & & & & 0,71 \\
\hline Strebla tonatiae Kessel, 1924 & & $\mathrm{X}$ & & & & & & 0,17 \\
\hline NYCTERIBIIDAE & & & & & & & & \\
\hline \multicolumn{9}{|l|}{ Subfamília Nicteribiinae } \\
\hline Basilia mimoni Teodor \& Peterson, 1964 & & $\mathrm{X}$ & & & & & & 0,17 \\
\hline Basilia sp. (travassoi cf.) & & $\mathrm{X}$ & & & & & & 0,17 \\
\hline Basilia sp. & & $\mathrm{X}$ & & & & & & 0,17 \\
\hline Total & 04 & 13 & 07 & 07 & 15 & 02 & 04 & \\
\hline
\end{tabular}

(Schinz, 1821), Phylloderma stenops Peters, 1865, Rhinophyla pumilio Peters, 1865, Artibeus cinereus (Gervais, 1856), Artibeus concolor Peters, 1865, Artibeus glaucus Thomas, 1893, Plathirrinus helleri (Peters, 1866), Uroderma bilobatum Peters, 1866, Myotis nigricans (Schinz, 1821) e Molossus rufus E. Geoffroy, 1805.

Apesar de este estudo ter sido realizado em poucas áreas e com coletas aleatórias, obteve-se como resultado uma grande riqueza de espécies. O número de estreblídeos registrados para o Maranhão só fica abaixo das 32 espécies de 12 gêneros registradas para o Paraná (Prevedello et al. 2005), sendo equivalente aos estudos de Guerrero (1994a, 1994b, 1995a, 1995b, 1996, 1997) que registraram 22 espécies de nove gêneros na região amazônica. Comparando ainda com outros estudos realizados a riqueza de espécie de estreblídeos registrada para o estado (23 espécies) já é superior às registradas para o Estado do Mato Grosso - 6 espécies (Pessôa \& Guimarães 1940), Minas Gerais - 12 espécies (Whitaker \& Mumford 1977; Komeno \& Linhares 1999; Azevedo \& Linardi 2002), Distrito Federal - 12 espécies (Coimbra et al. 1984, Graciolli \& Coelho
2001; Graciolli \& Aguiar 2002) e do Rio Grande do Sul - 11 espécies (Graciolli \& Rui 2001; Rui \& Graciolli 2005).

Já para os hospedeiros, das 33 espécies de morcegos registradas, 21 estavam infestadas (Tab. II), número este comparável ao encontrado em estudo sazonal realizado por Bertola et al. (2005) em uma reserva do estado de São Paulo, onde foram registradas das 22 espécies de morcegos registradas 17 infestadas.

Quanto à riqueza de espécies registradas por localidade, pudemos observar que o Quebra Pote e Bacabeira se sobressaíram em relação às demais, com 15 e 13 espécies respectivamente. Uma possível explicação para esse fato seria a heterogeneidade de habitats encontradas nessas localidades que contribuiria para o estabelecimento de uma assembléia mais complexa de morcegos e, por conseguinte de seus parasitos. Da mesma forma, o estádio de conservação da área contribui marcantemente para a riqueza de espécie, caso comprovado pelas sete espécies de moscas que só foram registradas em Bacabeira, localidade pouco impactada. Como também em Santa Inês foi registrado o menor número de 
Tabela II. Lista das espécies de morcegos capturados infestados e suas respectivas moscas ectoparasitas, registrados para o Estado do Maranhão.

\begin{tabular}{cc}
$\begin{array}{c}\text { Espécies de } \\
\text { morcegos }\end{array}$ & $\begin{array}{c}\text { Espécies de moscas } \\
\text { por hospedeiro }\end{array}$ \\
\hline EMBALLONURIDAE &
\end{tabular}

Saccopteryx leptura (Schreber, 1774)

NOCTILIONIDAE

Noctilio albiventris Desmarest, 1818 Paradyschiria parvula

PHYLLOSTOMIDAE

Artibeus lituratus (Olfers, 1818)

Aspidoptera phyllostomatis Megistopoda aranea Megistopoda aranea

Artibeus obscurus Schinz, 1821

Artibeus planirostris Spix, 1823

Aspidoptera phyllostomatis Trichobius joblingi

Paratrichobius longicrus

Carollia perspicillata (Linnaeus, 1758) Aspidoptera falcata

Speiseria ambigua Strebla guajiro

Trichobius costalimai

Trichobius joblingi

Mastoptera minuta

Trichobius joblingi Strebla diaemi

Trichobius diaemi

Carollia brevicauda Schinz, 1821

Desmodus rotundus E. Geoffroy, 1810 Glossophaga soricina Pallas, 1766

Lophostoma silvicolum d'Orbigny, 1836 Lophostoma brasiliense Peters, 1866

Trichobius parasicitus

Trichobius joblingi

Speiseria ambigua

Mastoptera minuta

Trichobius dugesii

Mastoptera minuta

Mastoptera minuta

Strebla tonatie

Lophostoma carrikeri J. A. Allen, 1910 Mastoptera minuta

Stizostrebla longirostris

Basilia mimoni

Mimon crenulatum E. Geoffroy, 1803

Micronycteris minuta Gervais, 1856

Phyllostomus discolor Wagner, 1843

Mastoptera minuta Strebla sp.

Stizostrebla longirostris Strebla hertigi

Trichobius costalimai

Trichobius dugesioides phyllostomus

Trichobioides perspicillatus

Phyllostomus hastatus Pallas, $1767 \quad$ Mastoptera minuta

Trichobius longipes

Aspidoptera falcata

Megistopoda proxima

Trichobius joblingi

Tonatia saurophila

Koopman \& Williams, 1951

VESPERTILIONIDAE

Myotis riparius Handley, 1960

Myotis albescens E. Geoffroy, 1806

Strebla galindoi

Basilia sp.

Basilia sp.

(travassoi ou sp. nov.)

espécies por ser a área menos complexa e mais impactada dentre as amostradas neste estudo.

Trichobius joblingi Wenzel, 1966 foi a espécie mais freqüente, representando $28,7 \%$ de todos os indivíduos coletados (Tab. I), isto possivelmente por esta espécie estar associada primariamente ao morcego Carollia perspicillata (Linnaeus, 1758), espécie comum em todos os ambientes amostrados, corroborando com as prevalências registradas para espécie nos estudos realizados no Estado de São Paulo $(42,5 \%)$ por Bertola et al. (2005), e Minas Gerais (66\%) por Komeno e Linhares (1999). Adicionalmente, esta espécie de mosca foi registrada na grande maioria das localidades, com exceção de Tufilândia onde este morcego coincidentemente não foi registrado infestado.

Com relação às associações parasito-hospedeiro, a espécie de morcego que apresentou o maior número de espécies diferentes de mosca foi $C$. perspicilatta (6 espécies); o registro de Speiseria ambigua Kessel, 1925, Strebla guajiro (Garcia \& Casal 1965) e Trichobius joblingi Wenzel, 1966 para este hospedeiro confirmam os estudos de Guerrero (1997). As outras três espécies encontradas neste hospedeiro, Aspidoptera falcata Wenzel, 1976, Mastoptera minuta (Costa Lima 1921) e Trichobius costalimai Guimarães, 1938, embora já registradas em estudos anteriores para este hospedeiro, podem ter suas ocorrências consideradas acidentais ou transitórias nesta região devido às suas raras ocorrências. Graciolli e Carvalho (2001) citam que esse tipo de associação pode estar relacionada a falhas na metodologia de coleta ou com a proximidade de abrigos utilizados por diferentes espécies de morcegos.

O morcego filostomídeo Phyllostomus discolor Wagner, 1843 foi o que apresentou a segunda maior riqueza de espécies de ectoparasitos (4 espécies) T. costalimai, Trichobioides perspicillatus (Pessôa \& Galvão 1937), Trichobius dugesioides phyllostomus Guerrero, 1998 e Strebla hertigi Wenzel 1966, sendo que as três últimas foram registradas apenas nesta espécie de hospedeiro (Tab. II).

A mosca ectoparasita registrada no maior número de espécies de morcegos foi Mastoptera minuta, parasitando os morcegos Glossophaga soricina Pallas, 1766, Micronycteris minuta Gervais, 1856, Lophostoma carrikeri (Allen, 1910), L. brasiliense (Peters, 1866) e Phyllostomus hastatus (Pallas, 1767) (Tab. II). Entretanto, pequenas variações morfológicas de M. minuta nestas espécies de hospedeiros foram descritas na Venezuela por Wenzel (1976) que seriam possivelmente espécies crípticas, fato que justifica a alta especificidade das associações entre parasita - hospedeiro, e cada uma das várias espécies utilizadas, porém até o momento essa taxonomia não foi oficializada (Dick \& Graciolli 2006).

As associações Artibeus planirostris Spix, 1823 parasitado por Paratrichobius longicrus (Miranda Ribeiro, 1907); Desmodus rotundus (E. Geoffroy, 1810) infestado por Trichobius parasiticus Gervais, 1844; Micronycteris minuta infestado por Mastoptera minuta; Noctilio albiventris Desmarest, 1818 parasitado por Paradyschiria parvula Falcoz, 1931; L. brasiliense parasitado por Strebla tonatie Kessel, 1924; Tonatia saurophila Koopman \& Williams, 1951 infestado por Strebla galindoi Wenzel, 1966; Sturnira lilium (E. Geoffroy, 1810) infestado por Aspidoptera falcata e Megistopoda proxima (Séguy, 1926), Aspidoptera phyllostomatis (Perty, 1833) em Phyllostomus discolor Wagner 
1843, e Megistopoda aranea (Coquillett, 1899) no morcego Artibeus obscurus (Schinz, 1821) confirmam os estudos de Guerrero (1995a, 1995b). As demais associações registradas na tabela II, inclusive para a família Nycteribiidae de Basilia mimoni Theodor \& Peterson, 1964 em Mimon crenulatum E. Geofroy, 1803, e do gênero Basilia sp. em Myotis riparius Handley, 1960 também já seriam esperadas.

O registro da mosca Stizostrebla longirostris Jobling, 1939 foi o segundo no Brasil, o primeiro depois de sua descrição em 1939 no Rio de Janeiro. Guerrero (1995b) cita a ocorrência dessa espécie para o Brasil, Bolívia, Venezuela e Colômbia comentando que em todos os registros essa mosca parasitava morcegos do gênero Tonatia sp. atual Lophostoma sp., e que seria enigmático a espécie-tipo ter sido registrada para o Rio de Janeiro, já que o gênero hospedeiro é amazônico. A falta de registros desta espécie no Brasil pode estar associada a uma combinação da falta de estudos com o grupo na região amazônica com a raridade de seus hospedeiros, que demonstraram nesse estudo serem bastante sensíveis a antropização sendo capturados apenas em manguezal primitivo no município de Bacabeira.

Foram ainda, registradas em várias localidades as associações de Trichobius costalimai no morcego Phyllostomus discolor, e de Mastoptera minuta no morcego Glossophaga soricina, o que poderia ser um indício de associação primária destas espécies para o Maranhão. Estes registros foram feitos pela primeira vez, fato que merece ser mais bem estudado.

O interior do Estado ainda encontra-se subamostrado. Foram realizadas coletas pontuais em apenas três municípios, Santa Inês e Tufilândia na porção central e Bacabeira no litoral central (Fig. 1), entretanto estas localidades apresentaram alta riqueza de espécies de moscas ectoparasitas, principalmente Bacabeira (13 espécies), demonstrando a grande biodiversidade do Estado e a necessidade de se conhecer todo esse potencial nos diversos biomas.

Adicionalmente, o baixo número de espécies da família Nycteribiidae registrado pode estar associada às baixas taxas de capturas de seus hospedeiros, que são morcegos principalmente das familias Vespertilionidae e Molossidae (Graciolli 2004).

\section{CONCLUSÃO}

O estado do Maranhão apresenta uma alta riqueza de espécies de moscas ectoparasitas de morcegos comparados aos outros estados do Brasil. A grande maioria das associações registradas seria esperada por já terem sido descritas na literatura, no entanto foram registradas as presenças de novas associações parasito-hospedeiro, merecendo maiores esforços de estudos. Adicionalmente, o maior esforço de amostragem dos morcegos no Estado poderá gerar novas perspectivas nos estudos de seus ectoparasitos bem como poderá aumentar a listagem de ocorrência dessas espécies.

Agradecimentos. Às fundamentais colaborações dos professores Tadeu Gomes de Oliveira (UEMA) e sua equipe na captura dos morcegos, e Gustavo Graciolli (UFMS) na identificação das moscas.

\section{REFERÊNCIAS}

Ab'saber, A. N. 1977. Os domínios morfoclimáticos na América do Sul. Geomorfologia 52: 1-21.

Azevedo, A. A. \& P. M. Linardi. 2002. Streblidae (Diptera) of Phyllostomidae bats from Minas Gerais, Brazil. Memórias do Instituto Oswaldo Cruz 97: 421-422.

Bertola, P. B.; C. C. Aires; S. E. Favorito; G. Graciolli; M. Amaku \& R. Pinto-Da-Rocha. 2005. Bat flies (Diptera: Streblidae, Nycteribiidae) parasitic on bats (Mammalia: Chiroptera) at Parque Estadual da Cantareira, São Paulo, Brazil: parasitism rates and host-parasite associations. Memórias do Instituto Oswaldo Cruz 100: 2532 .

Bredt, A. 1996. Morcegos em áreas urbanas e rurais: manual de manejo e controle. Fundação Nacional de Saúde. 117 p.

Burton K. L. \& M. D. Engstrom. 2001. Species diversity of bats (Mammalia: Chiroptera) in Iwokrama Forest, Guyana, and the Guianan subregion: implications for conservation. Biodiversity and Conservation 10: 613-657.

Coimbra-Jr., C. E. A.; L. R. Guimarães \& D. A. Mello. 1984. Ocorrência de Streblidae (Diptera: Pupipara) em morcegos capturados em regiões de cerrado do Brasil Central. Revista Brasileira de Entomologia 28: 547-550.

Dick, C. W. \& G. Graciolli. 2006. Checklist of world Streblidae (Diptera: Hippoboscoidea). National Science Foundation, 7p. Disponível em http://fm1.fieldmuseum.org/aa/Files/cdick/Streblidae_Checklist_ 2oct06.pdf

Fritz, G. N. 1983. Biology and ecology of bat flies (Diptera: Streblidae) on bats in the genus Carollia. Jornal de Medicina Entomológica 20: $1-10$.

Graciolli, G. \& L. S. Aguiar. 2002. Ocorrência de moscas ectoparasitas (Diptera, Streblidae e Nycteribiidae) de morcegos (Mammalia, Chiroptera) no Cerrado de Brasília, Distrito Federal, Brasil. Revista Brasileira de Zoologia 19: 177-181.

Graciolli, G. 2004. Nycteribiidae (Diptera: Hippoboscoidea) no sul do Brasil. Revista Brasileira de Zoologia 21: 971-985.

Graciolli, G. \& C. J. B. Carvalho. 2001. Moscas ectoparasitas (Diptera: Hippoboscoidea) de morcegos (Mammalia: Chiroptera) do estado do Paraná. II. Streblidae. Chave pictórica para gêneros e espécies. Revista Brasileira de Zoologia 18: 907-960.

Graciolli, G. \& D. C. Coelho. 2001. Streblidae (Diptera, Hippoboscoidea) sobre morcegos filostomídeos (Chiroptera, Phyllostomidae) em cavernas do Distrito Federal, Brasil. Revista Brasileira de Zoologia 18: 965-970.

Graciolli, G. \& C. W. Dick. 2006. Checklist of world Nycteribiidae (Diptera: Hippoboscoidea). National Science Foundation, 9p. Disponível em http://fm1.fieldmuseum.org/aa/Files/cdick/ Nycteribiidae_Checklist_2oct06.pdf.

Graciolli, G. \& P. M. Linardi. 2002. Some Streblidae and Nycteribiidae (Diptera: Hippoboscoidea) from Maracá Island, Roraima, Brazil. Memórias do Instituto Oswaldo Cruz 97: 139-141.

Graciolli, G \& A. M. Rui. 2001. Ocorrência de Streblidae (Diptera: Hippoboscoidea) em morcegos (Chiroptera, Phyllostomidae) no nordeste do Rio Grande do Sul, Brasil. Iheringia (Série Zoologia) 90: $85-92$.

Guerrero, R. 1993. Catalogo de los Streblidae (Diptera: Pupipara) parasitos de murcielagos (Mammalia: Chiroptera) del Nuevo Mundo. I. Clave para los gêneros y Nycterophilinae. Acta Biológica Venezuela 14: 61-75.

1994a. Catalogo de los Streblidae (Diptera: Pupipara) parasitos de murcielagos (Mammalia: Chiroptera) de Nuevo Mundo. II. Los grupos: pallidus, caecus, major, uniformis y longipes del gênero Trichobius Gervais, 1844. Acta Biológica Venezuela 15: $1-18$.

1994b. Catalogo de los Streblidae (Diptera Pupipara) parasitos de murciélagos (Mammalia:Chiroptera) del Nuevo Mundo. IV. Trichobiinae com alas desarrolladas. Boletim de Entomologia Venezuela 9: 161-192.

1995a. Catalogo de los Streblidae (Diptera: Pupipara) parasitos de murcielagos (Mammalia: Chiroptera) del 
Nuevo Mundo. III. Los grupos: dugesii, dunni y phyllostomae del gênero Trichobius Gervais, 1844. Acta Biológica Venezuela 15: $1-27$.

1995b. Catalogo de los Streblidae (Diptera: Pupipara) parasitos de murcielagos (Mammalia: Chiroptera) del Nuevo Mundo. V. Trichobiinae com alas reducidas o ausentes y miscelaneos. Boletim de Entomologia Venezuela 10: 135160.

1996. Streblidae (Diptera: Pupiara) parasitos de los murcielagos de Pakitza, Parque Nacional Manu (Peru). p. $627-$ 641. In: D. E. Wilson \& A. Sandoval (Eds). MANU: La biodiversidade del rureste del Peru. Washignton, Smithsonian Institution. $679 \mathrm{p}$.

1997. Catalogo de los Streblidae (Diptera: Pupipara) parasitos de murcielagos (Mammalia: Chiroptera) del Nuevo Mundo. VII. Lista de especies, hospedadores y paises. Acta Biologica Venezuela 17: 9-24.

Komeno C. A. \& A. X. Linhares. 1999. Bat flies parasitic some phyllostomid bats in Southeastern Brazil: parasitism rates and hostparasite relationships. Memórias do Instituto Oswaldo Cruz 94: $151-156$.

Maa. T. C. 1965. An interim world list of batflies (Diptera: Nycteribiidae and Streblidae). Journal of Medicine Entomological 1: 337 386.

Marinkelle, C. J. \& E. S. Grose. 1981. A list of ectoparasitas of Colombian bats. Revista de Biologia Tropical 29: 11-20.

Marshall, A. G. 1982. Ecology of Insets parasitic on bats. p. 369-401, In: Kunz, T. H. (ed) Ecology of bats. New York. Plenum Press. $533 \mathrm{p}$.

MMA. 2002. Áreas Prioritárias para a Biodiversidade. Brasília, DF. $463 \mathrm{p}$

Muniz, F. H. 2006. A vegetação de transição entre a Amazônia e o nordeste: diversidade e estrutura. Série Agroecologia - UEMA 2: $53-69$.
Pacheco, S. M., 2005. Técnicas de campo empregadas ao estudo de quirópteros. Caderno La Salle XI 2: 193-205.

Pereira, Y. N. O. \& J. M. M. Rebêlo. 2000. Espécies de Anopheles no município de Pinheiro (Maranhão), área endêmica de malária. Anais da Revista da Sociedade Brasileira de Zootecnia 33: 443450.

Pessôa, S. B. \& L. R. Guimarães. 1936. Notas sobre Streblidae (Diptera), com a descripção de um novo gênero e duas novas espécies. Anais da Faculdade de Medicina Universidade de São Paulo 12: 255-267.

Prevedello, J. A.; G. Graciolli \& C. J. B. Carvalho. 2005. A Fauna de dípteros (Streblidae e Nycteribiidae) ectoparasitos de morcegos (Chiroptera) do Estado do Paraná, Brasil: Composição, distribuição e áreas prioritárias para novos estudos. Biociências 13: 193209.

Rebêlo, J. M. M. \& J. M. L. Costa. 1996. Lista preliminar das espécies do gênero Lutzomyia Franá, 1924 (Phlebotominae, Psychodidae) do Estado do Maranhão-Brasil. Cadernos de Saúde Pública 12: 37-45.

Reis, N. dos; A. L. Peracchi; W. A. Pedro \& I. P. Lima. 2007. (Eds). Morcegos do Brasil. Londrina. 253 p.

Rui, A. M. \& G. Graciolli. 2005. Moscas ectoparasitas (Diptera, Streblidae) de morcegos (Chiroptera, Phyllostomidae) no sul do Brasil: associações hospedeiros-parasitas e taxas de infestação. Revista Brasileira de Zoologia, 22: 438-445.

Wenzel, R. L. 1970. Family Streblidae. In: N. Papavero (ed.). A catalogue of the Diptera south of the United States. Museo de Zoologia, São Paulo, Brazil, p. 100. 1-100. 25.

1976. The streblid batflies of Venezuela (Diptera: Streblidae). Brigham Young University Science Bulletin of Biology 20: 1-177.

Whitaker Jr., J. O. \& R. E. Mumford. 1977. Records of ectoparasitas from Brazilian mammals. Entomology News 88: 255-258. 